\title{
A FOCUSS-based Subspace Method for Blind Identification Robust to Order Overestimation
}

\author{
Wuqiang Zhou ${ }^{1, a}$ JingWei $\mathrm{Li}^{2, \mathrm{~b}}$ \\ ${ }^{2}$ Luoyang Institute of Science and Technology ,Henan, 471023 China \\ A,bzwq@lit.edu.cn
}

\begin{abstract}
Key words: Subspace method; FOCUSS algorithm; Augmented Lagrange function; Robustness to
\end{abstract} order overestimation

\begin{abstract}
A FOCUSS-based subspace method is proposed for blind channel identification, which is robust to channel order overestimation. Based on the sparsity of the desirable channel estimation to order overestimation, a weighted constraint is used to search the sparse solution, combining with the $l_{p}$ norm FOCUSS algorithm, then augmented Lagrange function is applied to improve the robustness of the FOCUSS algorithm. The simulation results show proposed algorithm owns better robustness to order overestimation than existing algorithms.
\end{abstract}

\section{Introduction}

Compared with the traditional method, wireless channel blind identification method dose not need to repeatedly send training sequence to obtain channel estimation, which saves precious bandwidth resources and channel capacity obviously increases. The blind identification algorithm based on second-order statistics represented by subspace algorithm has low complication and quick convergence, so that it causes universal attention. But defects of this method has bad order robustness, once channel order estimation is incorrect, algorithm performance will become increasingly aggravated.

The current order estimation algorithm mainly has information theory criterion algorithm ${ }^{[2]}$ and Liavas algorithm ${ }^{[3]}$ based on eigenvalue decomposition of receiving signal autocorrelation matrix. Recently, literature[4][5] also puts forward the new order estimation algorithm, but these algorithms inevitably need order to search optimization process and faces with higher calculation cost. In the real communication, order information of channel is not easily to realize precise estimation, while the upper boundary of order information can easily obtain from time-delay ${ }^{[5]}$ etc. In recent years, it appears a series of new algorithms having order robustness, it dose not need precise estimation of order and effective estimation of realizing channel on condition that it only knows channel upper boundary. Literature [6] puts forward one kind of order overestimation robust MCR (Modified Cross Relation) algorithm, but over-sampling enhancement will obviously increase calculation complication of algorithm, and this algorithm has weak performance under low noise-signal ratio. Algorithm based on CR obtains internal relation between solution space and real channel on condition of channel order overestimation, literature [7] puts forward order robust CR algorithm (this paper regards it as CR-FOCUSS Algorithm) based on FOCUSS (Foca1 Underdetermined System Solver), but the algorithm has weak robust.

This paper puts forward order robust subspace algorithm based on $l_{p}$ norm FOCUSS algorithm, on order overestimation, it firstly obtains solution space of $\boldsymbol{Q}$ matrix constructed by noise vector in SS algorithm and then it uses $l_{p}$ norm FOCUSS algorithm to obtain sparse solution so that it obtains channel estimation, meanwhile it introduces into Lagrange function to enhance robust of FOCUSS algorithm.

In the following, $E\{\cdot\}$ represents desired operation, $\operatorname{span}(\boldsymbol{A})$ indicates space generated by column space of $\boldsymbol{A},[\cdot]^{\mathrm{T}}, \quad[\cdot]^{\mathrm{H}}, \quad(\cdot)^{+}$respectively indicates transposition, conjugate transposition, Pseudo inverse operation of Moore-Penrose. 


\section{Algorithm Model and Space Algorithm}

The receiving terminal makes over-sampling on the received signal by single antenna of baud rate of $L$ times or uses $L$ antenna to receive by baud rate, so it can get SIMO channel. In the algorithm, the modeling equals to FIR channel of $M$ order.

$$
y_{i}(n)=\sum_{k=0}^{M} s(k) h_{i}(n-k)+v_{i}(n) \quad i=1, \ldots, L
$$

In the formula, $y_{i}(n)$ is the receiving signal of the $I$ sub-channel at time $n$, the parameter vector of sub-channel $\boldsymbol{h}_{i}=\left[h_{i}(0), \ldots, h_{i}(M)\right]^{\mathrm{T}},\{s(k)\}$ is the signal sequence has been sent off, $v_{i}(n)$ is the additive white Gaussian noise. The continual $N$ receiving signals of $L$ sub-channel can be represented as follows:

$$
\boldsymbol{y}(n)=\boldsymbol{H}_{N} \boldsymbol{s}(n)+\boldsymbol{v}(n)
$$

In the formula, the receiving signal vector of $L$ sub-channel is $y(n)=\left[\boldsymbol{y}_{1}^{T}(n), \ldots, \boldsymbol{y}_{L}^{T}(n)\right]^{T}$, of which, $\boldsymbol{y}_{i}(n)=\left[y_{i}(n), \cdots, y_{i}(n-N+1)\right]^{\mathrm{T}}$, sequence order of sending signal is $s(n)=[s(n), \ldots, s(n-N-M+1)]^{\mathrm{T}}$; noise vector is $\boldsymbol{v}(n)=\left[\boldsymbol{v}_{1}^{\mathrm{T}}(n), \ldots, \boldsymbol{v}_{L}^{\mathrm{T}}(n)\right]^{\mathrm{T}}$, of which, $\boldsymbol{v}_{i}(n)=\left[v_{i}(n), \ldots, v_{i}(n-N+1)\right]^{\mathrm{T}}$ represents the overlapped noise of the $i$ sub-channel, channel filtration matrix of $L N \times(M+N)$ dimension is $\boldsymbol{H}_{N}=\left[\boldsymbol{H}_{N}^{(1) \mathrm{T}}, \boldsymbol{H}_{N}^{(2) \mathrm{T}}, \ldots, \boldsymbol{H}_{N}^{(L) \mathrm{T}}\right]^{\mathrm{T}}$, of which, $\boldsymbol{H}_{N}^{(i)}$ has Toeplitz structure, the first line is $[h_{i}(0) h_{i}(1) \cdots h_{i}(M) \underbrace{0 \cdots 0}_{N-1}]$, the first row is $[h_{i}(0) \underbrace{0 \cdots 0}_{N-1}]^{\mathrm{T}}$ of $L N \times(M+1)$ sub-channel parameter vector is $\boldsymbol{h}=\left[\boldsymbol{h}_{1}^{\mathrm{T}}, \ldots, \boldsymbol{h}_{L}^{\mathrm{T}}\right]^{\mathrm{T}}$ 。

Get relevant matrix $\boldsymbol{R}_{y}=\mathrm{E}\left\{\boldsymbol{y}(n) \boldsymbol{y}(n)^{\mathrm{H}}\right\}$ for the observed and output $\mathbf{y}(n)$

$$
\boldsymbol{R}_{y}=\boldsymbol{H}_{N} \boldsymbol{R}_{s} \boldsymbol{H}_{N}{ }^{H}+\boldsymbol{R}_{v}
$$

In the formula, $\boldsymbol{R}_{s}=E\left\{\boldsymbol{s}(n) \boldsymbol{s}^{\mathrm{H}}(n)\right\}, \boldsymbol{R}_{v}=E\left\{\boldsymbol{v}(n) \boldsymbol{v}^{\mathrm{H}}(n)\right\}$. It makes eigenvalue decomposition for 对 $\boldsymbol{R}_{y}$.

$$
\boldsymbol{R}_{y}=\left[\boldsymbol{U}_{\mathbf{s}} \boldsymbol{U}_{v}\right] \operatorname{diag}\left(\lambda_{0}, \lambda_{1}, \cdots \lambda_{N+M-1}, \lambda_{N+M+1} \cdots \lambda_{L N-1}\right)\left[\begin{array}{c}
\boldsymbol{V}_{\mathbf{s}}^{\mathrm{H}} \\
\boldsymbol{V}_{v}^{\mathrm{H}}
\end{array}\right]
$$

In the formula, the corresponding left singular vector of $\lambda_{i}, 0 \leq i \leq N+M-1$ is $\boldsymbol{U}_{\mathrm{s}}=\left(\boldsymbol{u}_{0}, \cdots \boldsymbol{u}_{M+N-1}\right)$, of which, space generates into signal subspace, 其列空间张成信号子空间, the corresponding left singular vector of $\lambda_{i}, N+M \leq i \leq L N-1$ is $\boldsymbol{U}_{v}$, which generates into noise subspace. From the orthogonality relation and $\operatorname{span}\left\{\boldsymbol{U}_{\mathrm{s}}\right\}=\operatorname{span}\left\{\boldsymbol{H}_{N}\right\}$ of signal subspace and noise subspace, it can get the following:

$$
\boldsymbol{U}_{v}{ }^{\mathrm{H}} \boldsymbol{H}_{N}=0
$$

Command $\boldsymbol{G}_{i}(0 \leq i \leq L N-M-N-1)$ as column vector of $\boldsymbol{U}_{v}$, divide it into $L$ vectors combination $\boldsymbol{G}_{i, j}(j=1, \cdots L)$, which is $\boldsymbol{G}_{i}=\left[\boldsymbol{G}_{i, 1}^{\mathrm{T}}, \boldsymbol{G}_{i, 2}^{\mathrm{T}}, \cdots \boldsymbol{G}_{i, L}^{\mathrm{T}}\right]^{\mathrm{T}}, \quad \boldsymbol{g}_{i, j}$ of $(M+1) \times(M+N)$ dimensions is to use Toeplitz matrix formed by $\boldsymbol{G}_{i, j}$, of which, the first line is $[\boldsymbol{G}_{i, j}^{0} \boldsymbol{G}_{i, j}^{1} \cdots \boldsymbol{G}_{i, j}^{N-1} \underbrace{\boldsymbol{0} \cdots \boldsymbol{0}}_{M}]$, the first column is $[\boldsymbol{G}_{i, j}^{0} \underbrace{\mathbf{O} \cdots \boldsymbol{0} 0}_{M}]^{\mathrm{T}}$. Now it defines cost function:

$$
\mathbf{J}(\boldsymbol{h})=\left\|\boldsymbol{U}_{v}{ }^{\mathrm{H}} \boldsymbol{H}_{N}\right\|^{2}=\sum_{i=0}^{L N-M-N-1}\left\|\boldsymbol{G}_{i}^{\mathrm{H}} \boldsymbol{H}_{N}\right\|^{2}=\sum_{i=0}^{L N-M-N-1}\left\|\boldsymbol{h}^{\mathrm{H}} \boldsymbol{g}_{i}\right\|^{2}=\boldsymbol{h}^{\mathrm{H}} \boldsymbol{Q h}
$$

Of which, $\boldsymbol{g}_{i}=\left[\boldsymbol{g}_{i, 1}^{\mathrm{T}}, \boldsymbol{g}_{i, 2}^{\mathrm{T}} \cdots \boldsymbol{g}_{i, L}^{\mathrm{T}}\right]^{\mathrm{T}}, \boldsymbol{Q}=\sum_{i=0}^{L N-M-N-1} \boldsymbol{g}_{i} \boldsymbol{g}_{i}^{\mathrm{H}}$ 。

$$
\left\{\begin{array}{r}
\hat{\boldsymbol{h}}=\arg \min \{\mathrm{J}(\boldsymbol{h})\} \\
\text { s.t } \quad \boldsymbol{c}^{\mathrm{T}} \hat{\boldsymbol{h}}=1
\end{array}\right.
$$


Of which, $\boldsymbol{c}$ is random vector. SS algorithm channel estimation is the solution to demand formula.

\section{Order Robust Subspace Algorithm Based on FOCUSS}

In the subspace algorithm, when channel order overestimation that is the estimated order $\widehat{M}=m+1+M(m \geq 0)$, the null space corresponds to $\boldsymbol{Q}_{\widehat{M}}$ matrix is not un-dimensional but $m+1$ dimensions, which indicates that channel parameter can not be determined ${ }^{[9][10]}$ under one complex scalar fuzzy meaning. The ideal solution under condition of overestimation is as follows:

$$
\left\{\begin{array}{l}
\hat{\boldsymbol{h}}=\left[\hat{\boldsymbol{h}}_{1}, \cdots, \hat{\boldsymbol{h}}_{L}\right] \\
\hat{\boldsymbol{h}}_{i}=\alpha[\underbrace{0, \cdots 0, \boldsymbol{h}_{i}}_{\widehat{M}-M}]^{\mathrm{T}}=\alpha[\underbrace{0, \cdots 0, h_{i}(0), \cdots, h_{i}(M)}_{\widehat{M}+1}]^{\mathrm{T}} \\
i=1, \cdots, L
\end{array}\right.
$$

Of which, $\alpha$ is complex constant, in order to make $h_{i}(k)=0, k=M+1, \cdots \widehat{M}$, it can convert it into the following optimization problems:

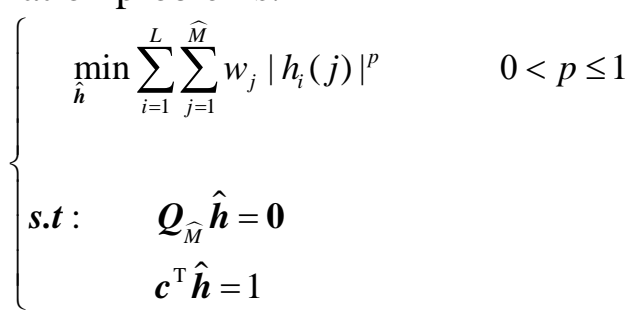

Of which, weight sequence $\left\{w_{j}\right\}_{j=1}^{\widehat{M}}$ is monotone decreasing, it can be chosen as exponential sequences $w_{j}=\mu^{j}, 0<\mu<1$. Because it is under overestimation condition, solution way of $\hat{\boldsymbol{h}}_{i}$ has sparse way. Now it defines $\boldsymbol{\mu}=\left[1, \mu, \cdots, \mu^{\widehat{M}}\right]^{\mathrm{T}}, \quad \boldsymbol{w}=\left[\boldsymbol{\mu}^{\mathrm{T}}, \cdots, \boldsymbol{\mu}^{\mathrm{T}}\right]^{\mathrm{T}}=\left[\tilde{w}_{1}, \cdots, \tilde{w}_{L(\widehat{M}+1)}\right]^{\mathrm{T}} \quad$, $\hat{\boldsymbol{h}}=\left[\hat{\boldsymbol{h}}_{1}, \cdots, \hat{\boldsymbol{h}}_{L}\right]^{\mathrm{T}}=\left[h_{1}, \cdots, h_{L(\widehat{M}+1)}\right]^{\mathrm{T}}, \quad \boldsymbol{y}=\left[\begin{array}{c}\boldsymbol{0}_{L(\widehat{M}+1)} \\ 1\end{array}\right], \quad \boldsymbol{A}=\left[\boldsymbol{Q}_{\widehat{M}}{ }^{\mathrm{T}} \boldsymbol{c}\right]^{\mathrm{T}}$, it can be simplified as follows:

$$
\left\{\begin{array}{l}
\min _{\hat{\boldsymbol{h}}} C(\hat{\boldsymbol{h}})=\min _{\hat{\boldsymbol{h}}} \sum_{j=1}^{L(\widehat{M}+1)} \widetilde{w}_{j}\left|h_{j}\right|^{p} \quad 0<p \leq 1 \\
\text { s.t }: \boldsymbol{A} \hat{\boldsymbol{h}}=\boldsymbol{y}
\end{array}\right.
$$

It uses Lagrange multiplier method to induce the following problems without restriction:

$$
\min _{\hat{h}} L(\hat{\boldsymbol{h}}, \boldsymbol{\lambda})=\min _{\hat{h}}\left\{\sum_{j=1}^{L(\widehat{M}+1)} \widetilde{w}_{j}\left|h_{j}\right|^{p}+\boldsymbol{\lambda}^{\mathrm{T}}(\boldsymbol{y}-\boldsymbol{A} \hat{\boldsymbol{h}})\right\}
$$

The above ways are similar to FOCUSS algorithm, it can get the following iteration form :

$$
\hat{\boldsymbol{h}}^{(k+1)}=\Pi^{-1}\left(\hat{\boldsymbol{h}}^{(k)}\right) \cdot \boldsymbol{A}^{\mathrm{T}}\left(\boldsymbol{A} \cdot \Pi^{-1}\left(\hat{\boldsymbol{h}}^{(k)}\right) \cdot \boldsymbol{A}^{\mathrm{T}}\right)^{-1} \boldsymbol{y}
$$

Of which, $\Pi^{-1}(\hat{\boldsymbol{h}})=\operatorname{diag}\left(\left|\hat{h}_{1}\right|^{2-p} / \widetilde{w}_{1}, \cdots,\left|\hat{h}_{L(\widehat{M}+1)}\right|^{2-p} / \widetilde{w}_{L(\widehat{M}+1)}\right)$ is weighted matrix, the formula is the signal reconstruction recurrence formula. FOCUSS method makes solution vector energy localization so that it can get solutions with sparse way by gradual iteration. The diagonal matrix in the formula $\Pi\left(\hat{\boldsymbol{h}}^{(k)}\right)$ will become increasingly sparse with algorithm iteration, the pathological degree of matrix $\boldsymbol{A} \cdot \Pi^{-1}\left(\hat{\boldsymbol{h}}^{(k)}\right) \cdot \boldsymbol{A}^{\mathrm{T}}$ become worsen, while for estimation precision of inverse matrix $\Pi\left(\hat{\boldsymbol{h}}^{(k)}\right)$ has big influence on stability and noise resistance performance of FOCUSS algorithm.

This paper introduces into augmentation Lagrange function method to get optimization model, augmentation Lagrange function method constructs precise penalty function, proper choice on augmentation factor can use little iteration times to make cost function converge to the optimal solution ${ }^{[11][12]}$ of original problem. It can construct sequence and problems without restriction. 


$$
\min _{\widehat{\mathrm{h}}} L\left(\hat{\boldsymbol{h}}, \lambda, \beta_{k}\right)=\min _{\hat{\mathrm{h}}}\left\{\sum_{j=1}^{L(\widehat{M}+1)} \widetilde{w}_{j}\left|h_{j}\right|^{p}+\lambda^{\mathrm{T}}(\boldsymbol{y}-\boldsymbol{A} \hat{\boldsymbol{h}})+\frac{\beta_{k}}{2}\|\boldsymbol{y}-\boldsymbol{A} \hat{\boldsymbol{h}}\|_{2}^{2}\right\}
$$

Introduction of augmentation factor can reduce noise to certain extent.

$$
\frac{\partial L\left(\hat{\boldsymbol{h}}, \lambda, \beta_{k}\right)}{\partial \hat{\boldsymbol{h}}}=p \cdot \Pi(\hat{\boldsymbol{h}}) \cdot \hat{\boldsymbol{h}}+\boldsymbol{A}^{\mathrm{T}} \lambda+\beta_{k} \boldsymbol{A}^{\mathrm{T}}(\boldsymbol{y}-\boldsymbol{A} \hat{\boldsymbol{h}})=\mathbf{0}
$$

It can be known by mathematical derivation:

$$
\hat{\boldsymbol{h}}=\frac{1}{\beta_{k}}\left(\frac{p}{\beta_{k}} \cdot \Pi(\hat{\boldsymbol{h}})+\boldsymbol{A}^{\mathrm{T}} \boldsymbol{A}\right)^{-1} \boldsymbol{A}^{\mathrm{T}} \cdot\left(\beta_{k} \boldsymbol{y}-\boldsymbol{\lambda}\right)
$$

It knows the following iteration form.

$$
\hat{\boldsymbol{h}}^{(k+1)}=\frac{1}{\beta_{k}} \Pi^{-1}\left(\hat{\boldsymbol{h}}^{(k)}\right) \cdot \boldsymbol{A}^{\mathrm{T}}\left(\boldsymbol{A} \cdot \Pi^{-1}\left(\hat{\boldsymbol{h}}^{(k)}\right) \cdot \boldsymbol{A}^{\mathrm{T}}+\frac{p}{\beta_{k}} \boldsymbol{I}\right)^{-1}\left(\beta_{k} \boldsymbol{y}-\boldsymbol{\lambda}_{k}\right)
$$

From the formula it can seen that matrix $A \cdot \Pi^{-1}\left(\hat{\boldsymbol{h}}^{(k)}\right) \cdot \boldsymbol{A}^{\mathrm{T}}+\frac{p}{\beta_{k}} \boldsymbol{I}$ is one symmetrical, positive and invertible matrix, augmentation factor $\beta_{k}$ only needs to choose one incremental and limited arithmetic number to guarantee formula converged to the optimal solution, it can be defined as follows:

$$
\beta_{k+1}=\beta_{0}\left(1+k^{a}\right) \quad a>0
$$

In the formula, $\beta_{0}$ and $a$ are all constant, Lagrange multiplier vector $\lambda$ can be obtained through iteration form:

$$
\lambda_{k+1}=\lambda_{k}+\beta_{k}\left(A \hat{\boldsymbol{h}}^{(k)}-\boldsymbol{y}\right)
$$

Matrix $\boldsymbol{A} \cdot \Pi^{-1}\left(\hat{\boldsymbol{h}}^{(k)}\right) \cdot \boldsymbol{A}^{\mathrm{T}}+\frac{p}{\beta_{k}} \boldsymbol{I}$ still keeps inevitable and avoids appearance of ill-conditioned matrix with the iteration updating of augmentation factor, which guarantees the precision of channel recovery.

\section{Simulation Analysis}

Test channel adopts one SIMO channel with $L=4, M=4$ in literature [1], channel parameter is indicated by table 1 . In the simulation, the emission signals are all evenly distributed 16QAM signal, the overlapped noise is white Gaussian noise, WGN. The evaluation of identification result adopts Normalized Root Mean Square Projection Misalignment, NRMSPM, it is defined as follows:

$$
\operatorname{NRMSPM}(\mathrm{dB}) \Delta \frac{1}{N_{m}} \sum_{i=1}^{N_{m}} \log _{10}\left(1-\left\|\frac{\mathbf{h}^{{ }^{* T}} \mathbf{h}_{i}^{*}}{\left\|\mathbf{h}^{{ }^{* T}}\right\|\left\|\mathbf{h}_{i}^{*}\right\|}\right\|^{2}\right)
$$

Of which, in the test, it sets Monte Carlo test times $N_{m}=50$, command $\mathbf{h}_{i}$ as identification result of the $\mathrm{i}$ times, $\mathbf{h}$ indicates the real channel, $\mathbf{h}_{i}$ and $\mathbf{h}$ need to implement corresponding zero-fill into $\mathbf{h}_{i}^{*}$ and $\mathbf{h}^{*}$ so that keeps consistence in length. In the test, $\beta_{0}=5, a=0.2, p=1$, $\Delta M=\widehat{M}-M \in[0,10], \quad N=20$.

Table 1 Channel parameter

\begin{tabular}{cccccc}
\hline$n$ & 0 & 1 & 2 & 3 & 4 \\
\hline$h_{1}(n)$ & $-0.049+0.359 \mathrm{i}$ & $0.482-0.569 \mathrm{i}$ & $-0.556+0.587 \mathrm{i}$ & 1 & $-0.171+0.061 \mathrm{i}$ \\
$h_{2}(n)$ & $0.443-0.036 \mathrm{i}$ & 1 & $0.921-0.194 \mathrm{i}$ & $0.189-0.208 \mathrm{i}$ & $-0.087-0.054 \mathrm{i}$ \\
$h_{3}(n)$ & $-0.221-0.322 \mathrm{i}$ & $-0.199+0.918 \mathrm{i}$ & 1 & $-0.284-0.524 \mathrm{i}$ & $0.136-0.19 \mathrm{i}$ \\
$h_{4}(n)$ & $0.417+0.030 \mathrm{i}$ & 1 & $0.873+0.145 \mathrm{i}$ & $0.285+0.309 \mathrm{i}$ & $-0.049+0.161 \mathrm{i}$ \\
\hline
\end{tabular}

\section{A. Comparison test between SS-FOCUS algorithm and CR-FOCUSS algorithm}

In this test, it regards channel order overestimation as $\widehat{M}=7$, which is $\Delta M=3$, the weighted 
factor in SS-FOCUS algorithm $\mu=0.003$. Figure 1 and figure 3 are SS-FOCUSS algorithm when noise-signal ratio is $20 \mathrm{~dB}$ and $70 \mathrm{~dB}$, channel response diagram of CR-FOCUSS and MCR. Because CR-FOCUSS, MCR algorithm involve with option of $\mu$ in weighted factor, all has been taken proper value in the simulation test. From diagram 1 it can be seen that CR-FOCUSS and MCR algorithm are ineffective, while identification result of SS-FOCUSS algorithm are well fit with real channel, the overestimation part tends to be zero. Diagram 2 uses channel estimation obtained by SS-FOCUS algorithm from diagram 1 into zero forcing equalization and gets signal constellation, it only has certain phase fuzzy and amplitude change. From diagram 3, it can be seen that 3 kinds of algorithms under noise-signal ratio of $70 \mathrm{~dB}$ can well fit the channel, and performance of SS-FOCUS algorithm and CR-FOCUSS algorithm is superior to that of MCR algorithm.
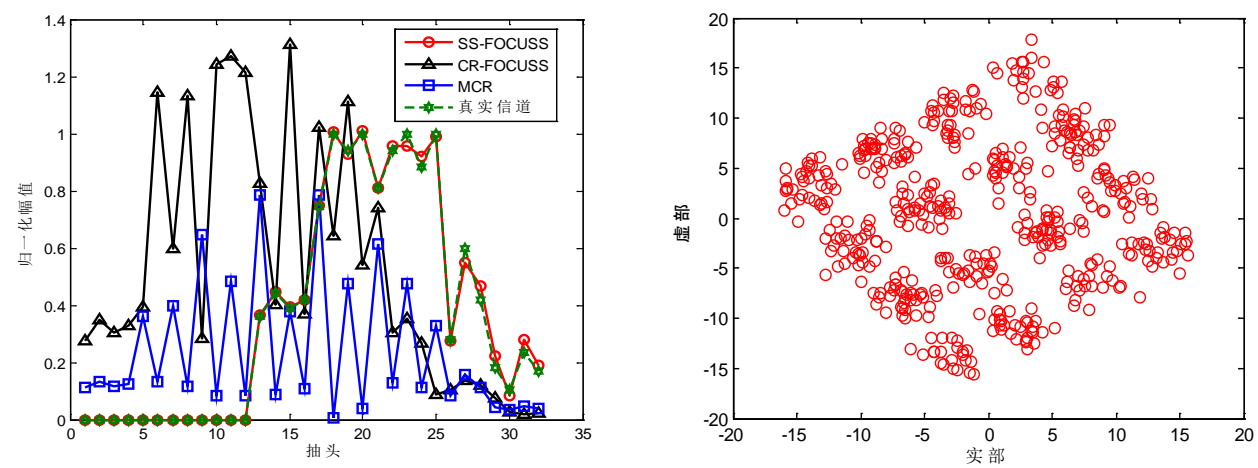

Figure 1 Estimation channel response diagram of 20dBFigure 2 Balance result of SS-FOCUS algorithm
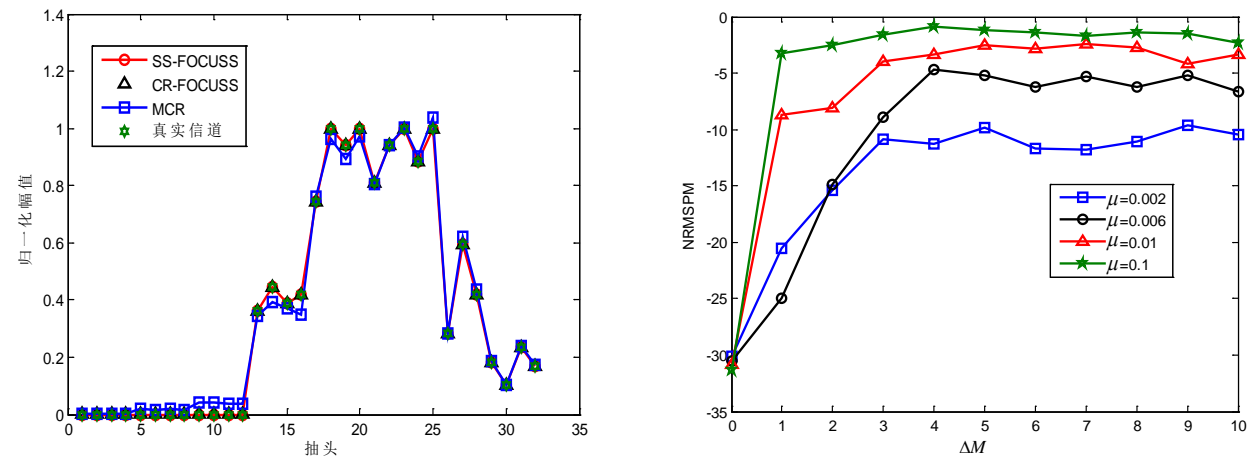

Figure 3 Estimation channel response diagram of 70dB Figure 4 Change curve $(\mathrm{SNR}=20 \mathrm{~dB})$ of $\mathrm{NRMSPM}-\Delta M$

\section{B. Change test of NRMSPM with over-order $\Delta M$ and $\mu$}

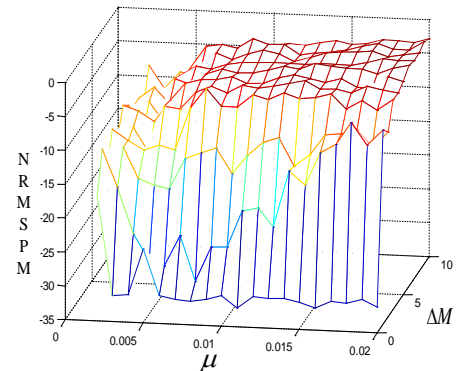

(a) $\mathrm{SNR}=20 \mathrm{~dB}$

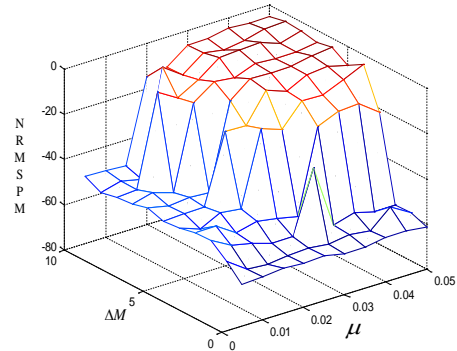

(b) $\mathrm{SNR}=50 \mathrm{~dB}$

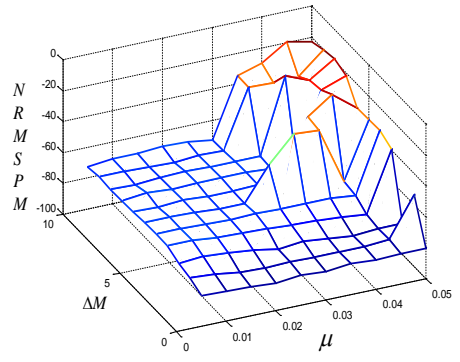

(c) $\mathrm{SNR}=70 \mathrm{~dB}$

Figure 5 Changes of NRMSPM wit h $\mu$ and $\Delta M$ under different signal-noise ratio

In this chapter, under the given condition of test and research, change condition of NRMSPM 
with $\Delta M$ and $\mu$. Figure 4 is the change diagram with $\Delta M$ when $\mathrm{SNR}=20 \mathrm{~dB}$, when $\Delta M(\Delta M \leq 2)$ is smaller, the value of $\mathrm{NRMSPM}$ is smaller than $-15 \mathrm{~dB}$, when $\Delta M$ is bigger ( $3 \leq \Delta M \leq 10$ ), NRSPM smoothly changes and value is less than $-10 \mathrm{~dB}$, which indicates that SS-FOCUSS algorithm order robust is very strong, while MCR algorithm and CR-FOCUSS algorithm has limitations in anti-order overestimation capacity, usually it has good performance under high signal-noise ratio and overestimation order is little. From figure 4 it can be seen that with the increase of $\mu$, NRMSPM of SS-FOCUSS algorithm gradually moves upwards with the change curve of $\Delta M$, algorithm is bad when $\mu=0.1$, which indicates that choice of $\mu$ is very important.

Figure 5is the change diagram of NRMSPM with $\mu$ and $\Delta M$ under different signal-noise ratio. Figure 5(b)is under condition that SNR $=50 \mathrm{~dB}$, when $0.005 \leq \mu \leq 0.02$, NRMSPM $<-40 \mathrm{~dB}$; when figure 5(c)is under condition of $\mathrm{SNR}=70 \mathrm{dBit}$ can be seen that when $0.005 \leq \mu \leq 0.035$, NRMSPM $<-50 \mathrm{~dB}$, diagram appears relatively smooth low land of $L$ type(it is called by comparing with the high land in the diagram), compares with the corresponding $L$ area in figure 5(b), the square increases. The above results indicate that with the increase of signal-noise ratio, choice on $\mu$ can be properly enlarged, meanwhile, the smaller value of $\mu$ is always one good choice.

\section{Conclusion}

This paper puts forward one kin of order robust subspace algorithm based on FOCUSS, the ideal sparse characteristic of algorithm based on subspace on order overestimation, it combines with $l_{p}$ norm FOCUSS algorithm to get sparse solution on the given exponential weighting restriction, meanwhile it introduces into augmentation Lagrange function method to increase robust and algorithm precision of FOCSS algorithm. The algorithm in this paper can adopt reducing subspace algorithm in the literature [14] to calculate complication, while the convergence speed of accelerating FOCUSS algorithm can apply algorithm in the literature [15]. The theoretical analysis and simulation indicates that the performance of SS-FOCUSS put forward by this paper is obviously superior to the current CR-FOCUSS algorithm and MCR algorithm,, and it can choose one small, proper weight factor $\mu$ to adapt to the large overestimation order range, so it has stronger order robust.

\section{References}

[1] Moulines E, Duhamel P, Cardoso J F, et al. Subspace-methods for the Blind Identification of Multi-channel FIR Filters [J]. IEEE Transactions on Signal Processing, 1995, 43(2): 516-525

[2] Ridder F D, Pintelen R, Schoukens J,et al. Modified AIC and MDL model selection criteria for short data records. [J]. IEEE Transactions on Instrument and Measurement,2005, 54(1):144-150

[3] Liavas A P, Regalia P. On the behavior of information theoretic criteria for model order selection [J]. IEEE Transactions on Signal Processing,2001,49(8):1689-1695.

[4] 代松银,袁嗣杰,董书攀. 基于子空间分解的信道阶数估计算法[J]. 电子学报, 2010,38(6): 1245-1248.

[5] Karakutuk S, Tuncer $\mathrm{T}$ E. Channel matrix recursion for blind effective channel order estimation.[J]. IEEE Transactions on Signal Processing, 2011, 59(4): 1642-1653

[6] Shi M, Yi Q M. An Efficient Blind SIMO Channel Identification Algorithm Via Eigenvalue Decomposition[J]. Lecture Notes in Control and Information Sciences, 2006,344(1):41-47.

[7] He Z S, Cichocki A. Robust channel identification using FOCUSS method [J]. Advance in Neural Network Research and Application,2010,67(1):471-477.

[8] Rao B D, Kreutz D. An affine scaling methodology for best basis selection[J]. IEEE 
Transactions on Signal Processing, 1999, 47(1):641-654.

[9] Meraim K A, Cardoso J F. On subspace methods for blind identification of single-input multiple-output FIR system.[J]. IEEE Transactions on Signal Processing, 1997, 45(1):42-55.

[10]Schmid D , Enzner G. Cross-relation-based blind SIMO identifiability in the presence of near-common zeros and noise[J]. IEEE Transactions on Signal Processing, 2012, 60(1):60-72.

[11]Magnusr R. Multiplier and gradientmethods [C].the Second International Conference on Computing Methods in Optimization Problems. San Remo,Italy,1968.

[12]杨俊杰,刘海林.增广 lagrange 函数优化算法在稀疏信号重构问题中的应用[J]. 计算机科学, 2011,38(9): 193-196.

[13]Dennis R. Morgan, Jacob Benesty, M. Mohan Sondhi. On the evaluation of estimated impulse responses[J]. IEEE Signal Processing Letters,1998,5(7):174-176.

[14]Koji $\mathrm{H}$ and Hideaki S. Blind subspace-based channel estimation using the EM algorithm[C].IEEE International Conference on Acoustics, Speech and Signal Processing, Taipei ,2009.2797-2800

[15]He Z S, Ciehoeki H A, Zdunek R, et al. Improved FOCUSS method with conjugate gradient iterations[J]. IEEE Transactions on Signal Processing,2009,57(1):399-404 